\title{
EXERCISE DOES NOT AFFECT STIFFNESS AND MINERALISATION OF THIRD METACARPAL CONDYLAR SUBARTICULAR CALCIFIED TISSUES IN 2 YEAR OLD THOROUGHBRED RACEHORSES
}

\author{
${ }^{1,2}$ V.L. Ferguson, ${ }^{2}$ A.J.Bushby, ${ }^{3}$ E.C. Firth, ${ }^{4}$ P.G.T. Howell and ${ }^{4 *}$ A.Boyde \\ ${ }^{1}$ Department of Mechanical Engineering, UCB 427, University of Colorado, Boulder, CO 80309, USA. \\ ${ }^{2}$ Department of Materials, Queen Mary, University of London, Mile End Rd, London E1, UK. \\ ${ }^{3}$ Massey Equine, Institute of Veterinary, Animal and Biomedical Sciences, and National Research Centre for Growth \\ and Development, Massey University, Palmerston North, New Zealand. \\ ${ }^{4}$ Barts and The London School of Medicine and Dentistry, Queen Mary, University of London, London E1 1BB, \\ UK.
}

\begin{abstract}
Impact exercise has a profound effect in increasing volumetric density of epiphyseal bone, as clearly shown in 2 year old thoroughbred racehorses from which we derived the tissue studied in the present investigation. Here, we asked the question whether the fabric-level properties of the mineralised tissues immediately below hyaline articular cartilage which transmit the extra loads are themselves altered in consequence.

We therefore studied the nanoindentation elastic modulus and its relationship to the concentration of mineral determined by quantitative backscattered electron imaging in the heavily loaded palmar medial and lateral condyles of the distal third metacarpal bone (Mc3) of 4 untrained and 4 trained 2-year old Thoroughbred racehorses.

We found no difference between trained and untrained horses in either subchondral bone or calcified cartilage in the mean stiffness or mineral content or their correlation. Thus neither articular calcified cartilage nor the immediately adjacent subchondral bone were affected by exercise, even though they transmitted the higher load associated with athletic training through to the deeper bone, which itself responded floridly to exercise. Under the circumstances of this experiment and at least in the very small regions studied, therefore, the structure of these two tissues was apparently optimised to function.
\end{abstract}

Keywords: calcified cartilage, subchondral bone, exercise, mechanical properties, mineralisation

\footnotetext{
*Address for correspondence:

Professor Alan Boyde,

Biophysics, Oral Growth and Development,

Barts and The London School of Medicine and Dentistry, Queen Mary, University of London,

Dental Institute, New Road, London E1 1BB, UK.

Telephone number +442078828653

E-mail: a.boyde@qmul.ac.uk
}

\section{Introduction}

The structure and function of articular calcified cartilage has been studied much less than the adjacent hyaline articular cartilage and subchondral bone layers, perhaps because there are difficulties in examining such a thin hard tissue layer interposed between two tissues of disparate character (Ferguson et al., 2003; Gupta et al., 2005). The effects of growth and of imposed exercise have rarely been studied separately in these tissues, yet athletic training of both horses and humans begins while growth is still occurring. Tissue abnormalities, variations in joint conformation and possibly clinical signs of joint disease are factors which complicate our understanding of the relationships between tissue development, lesions and exercise.

The Massey University Grass Exercise Study (MUGES) was established to assess tissue responses in young thoroughbred horses in early training, before the heavier cyclic exercise loads associated with training and racing at high intensity or with a prolonged athletic career in older racehorses. It provided material in which we could compare articular cartilage and bone in pasture-raised horses, one group of which was trained for 13 weeks before tissue examination and comparison with an untrained group (Firth et al., 2004, 2005; Firth and Rogers, 2005).

Study of the distal part of the third metacarpal bone (Mc3) in these two groups of animals showed large and obvious differences in bone tissue in the trained group. In particular, there was a marked densification due to an increase in the volume fraction occupied by bone (i.e., a decrease in porosity, or decrease in marrow space volume) in the palmar portion of the condyles by the formation of new bone within prior fatty marrow space (Boyde and Firth, 2005). The regions where new bone formed most abundantly were deep to those where mechanical loading at the joint surface appears to be greatest. This extra load is transmitted through to the deep epiphyseal domain bone layers via the soft, hyaline cartilage, its deep calcified layer and the immediately proximate subchondral bone. However, it is not known whether changes also occur in the mechanical properties of calcified cartilage and subchondral bone.

The purpose of the present study was to determine whether there were detectable between-horse differences in the nature of the articular calcified cartilage and 
subchondral bone when assessed by methods which test stiffness modulus and mineralisation density at micron scale resolution. We have previously established methods to correlate quantitative backscattered electron (qBSE) measurements of tissue mineralisation with nanoindentation measurements of tissue modulus to examine articular calcified cartilage and subchondral bone in the adult human femoral head (Ferguson et al., 2003). Here, we sought to examine the range of variation in composition and mechanical response of calcified cartilage and subchondral bone in the distal third metacarpal bone in a sub-group of young horses from the MUGES study, and to determine if there was a significant relationship between exercise level, mechanical loading by nano-indentation, and tissue properties in the condylar sites where loading is high, as evidenced by maximal changes in epiphyseal bone post-exercise in the heavily loaded palmar condylar regions (Firth et al., 2005; Boyde and Firth, 2005).

\section{Materials and Methods}

Pasture-raised, rising 2 year old thoroughbred fillies were trained with a rider on grass and sand racetracks for 13 or 14 weeks, and were housed in stalls overnight and in dirt yards during the day. Untrained horses remained in pairs in $25 \times 15 \mathrm{~m}$ grass enclosures in which they could exercise at will (Firth et al., 2004). Following euthanasia, the distal right $\mathrm{Mc} 3$ was frozen at $-20^{\circ} \mathrm{C}$ until preparation for examination. Samples from 4 untrained horses and 4 trained MUGES horses were used in this study.

The trained horses 14, 50 and 55 all galloped $4450 \mathrm{~m}$ (7 gallops in 4 weeks, at a mean velocity of $14.62 \mathrm{~m} / \mathrm{s}$ ), and had run very similar distances during the previous 9 weeks of early training. Although trained, Horse 60 refused to gallop from week 10, but had covered Phase 1 and 2 like the other 3 subjects, at canter velocities of 7.54-8.9 $\mathrm{m} / \mathrm{s} 6$ days per week for $\sim 9$ weeks. Details of workload of each individual are available from Firth et al., (2004) and Rogers and Firth (2004). The untrained horses could neither canter nor gallop.

The methods used to study calcified cartilage and the immediately adjacent subchondral bone have been detailed by Boyde and Firth (2005). Of relevance to this study, the epiphysis was sawn with a water cooled, slow-speed diamond saw (LabCut; DR Bennett Ltd, London, UK) to produce slices in the palmar-oblique plane, perpendicular to the joint surface at $30-35^{\circ}$ to the medio-lateral frontal plane, so as to pass through those regions of the condyles which showed the greatest densification in response to exercise (Firth et al., 2005).

\section{qBSE SEM}

The slices were embedded in PMMA, the resulting blocks micro-milled, carbon-coated and scanned using quantitative backscattered electron imaging (qBSE) in a Zeiss DSM962 automated digital SEM with a Kontron IBAS external computer control (both purchased from Zeiss UK Ltd, Welwyn Garden City, Herts, UK) with an annular solid state BSE detector (from KE Electronics Ltd, Toft, Cambs, UK).
Using the montaged qBSE images, regions were chosen for nanoindentation testing on the medial and lateral condyles at $40 \%$ of the distance from the abaxial border of the slice to the junction of ridge and condyle (Figure 1).

\section{Nanoindentation}

The PMMA blocks were fastened to steel mounts using cyanoacrylate adhesive. Regions of interest were tested using a UMIS 2000 nanoindentation system (CSIRO, Sydney, Australia) using a $5 \mu \mathrm{m}$ radius spherical geometry diamond indenter tip to a maximum load of $15 \mathrm{mN}$. The tip shape and frame stiffness were calibrated using a multiple reference material method (Bushby and Jennett, 2001). Each indentation test consisted of 40 load increments, unloading to $75 \%$ of each load between increments where the plane strain elastic indentation modulus $(E)$ was calculated as a function of contact depth (Field and Swain, 1993; Bushby, 2001) for each load partial-unload data pair, and a mean value of modulus was derived for each indentation site from the 20 deepest increments. Arrays of indents at $20 \mu \mathrm{m}$ spacing, ranging in width from 5-10 indents $(80-180 \mu \mathrm{m})$, were positioned to span from hyaline through calcified cartilage to bone.

\section{Matching sites of qBSE and nanoindentation measurements}

After indentation, the array sites were found by location in the original lower resolution montage images. This was aided by the use of deep marker indents at each end of the fields of interest. Generally, the indent sites could not be seen in the compositional ('A+B') mode. Each field was rescanned at high resolution (nominal 150' magnification, $594 \mu \mathrm{m}$ and 2048 pixels wide, $20 \mathrm{kV}$ accelerating voltage, $0.5 \mathrm{nA}$ probe current, $17 \mathrm{~mm}$ working distance, $11 \mathrm{~mm}$ detector to sample distance. Each field was scanned twice, first in compositional (' $\mathrm{A}+\mathrm{B}$ ') mode by summing the output of all four BSE detector quadrants, and then in topographic ('A-B') mode using the difference between opposing detector segment pairs to permit the exact location of the indent sites (Ferguson et al., 2003).

Halogenated dimethacrylate polymers were used as standards against which to measure relative electron backscattered intensity. Measured grey scale values were rescaled such that that from the monobrominated compound was set to zero, and that from the monoiodinated compound to 255 (Howell and Boyde, 1998a,b; Boyde and Firth, 2005). The topographic images were used to derive a binary image mask with appropriately spaced patches that lay over each separate indentation site. This image was aligned with large, easily visible marker indents at the array boundaries. The binary mask was overlain on the corresponding compositional image (Fig.1).

Each indent site was identified from the compositional qBSE image as hyaline or calcified cartilage, bone, bonebone cement line, bone to calcified cartilage cement line, osteocyte lacuna, chondrocyte lacuna, or marrow space. Sites classified as other than calcified cartilage or bone were excluded. To avoid any influence from neighbouring tissues or defects, measurements from sites within $10 \mu \mathrm{m}$ of cracks, edges, cement lines, lacunae or other voids were also excluded. The mineral concentration at each site [mean 
grey level from $\sim 220$ pixel area] was derived from the mean qBSE grey level using SigmaScan Pro 5 software (SPSS Inc, Chicago, Illinois 60606, USA). The mineral volume fraction $(\mathrm{MVF} \%)$ at each voxel within the embedded tissue was calculated from the qBSE grey levels by comparison with electron backscattering from standard reference materials (Howell and Boyde, 1998a,b).

\section{Validation}

The qBSE method has been validated previously by repeated measurements of BSE spectra from a set of elements and compounds of known composition. The spectra were recorded under different operating conditions and repeated on different occasions and compared after normalizing to the $\mathrm{MBr}(0)$ and MI (255) standards. The differences in the modal values varied by 1-2 grey levels in 256 , i.e. $\sim 1 \%$.

It is important to note that the halogenated dimethacrylate polymer standards, unlike either ionic or metallic crystalline materials, have no long range order and there are no electron channelling (diffraction) effects. Pure metals and ionic crystals should not be used as standards in this work, since different areas of the same standard give different signal levels according to the degree of channelling, and broad spectral peaks for $Z$ vs. observed BSE intensity are found experimentally. On the other hand, sharply defined peaks are derived in experiments with halogenated polymers (Howell et al., 2001).

The validation of the nano-indentation technique was achieved in accordance with the standard ISO 14577 for instrument calibration and indenter tip shape determination using certified reference materials. The total uncertainty in the measurement of modulus was $<1.5 \%$ of the measured value.

\section{Statistical Analysis}

The data set presented here consists of 1023 indent sites on calcified cartilage and 1184 on bone.

A two way analysis of variance (ANOVA) with a confidence level of 0.05 and a Tukey's post-hoc analysis was used to test for differences due to exercise and tissue type in the two groups $(n=4)$ of horses.

For both bone and calcified cartilage, there was no significant difference in mineralisation or elastic modulus between the medial and lateral condylar sites, which were therefore pooled for analysis.

\section{Results and Discussion}

We present the first data correlating matrix mineralisation and nano-indentation modulus for calcified cartilage and the immediately adjacent subchondral bone in large young mammals that had undertaken training exercise. In interpreting our data, it should be borne in mind that the tissue layer we have treated as subchondral bone is within $\sim 250$ micrometers of calcified articular cartilage. Most prior literature - with the exception of our previous study on the aged human femoral head (Ferguson et al., 2003) and Gupta et al.'s (2005) study of human patellae deals with a much thicker layer of bone. The thin layer of

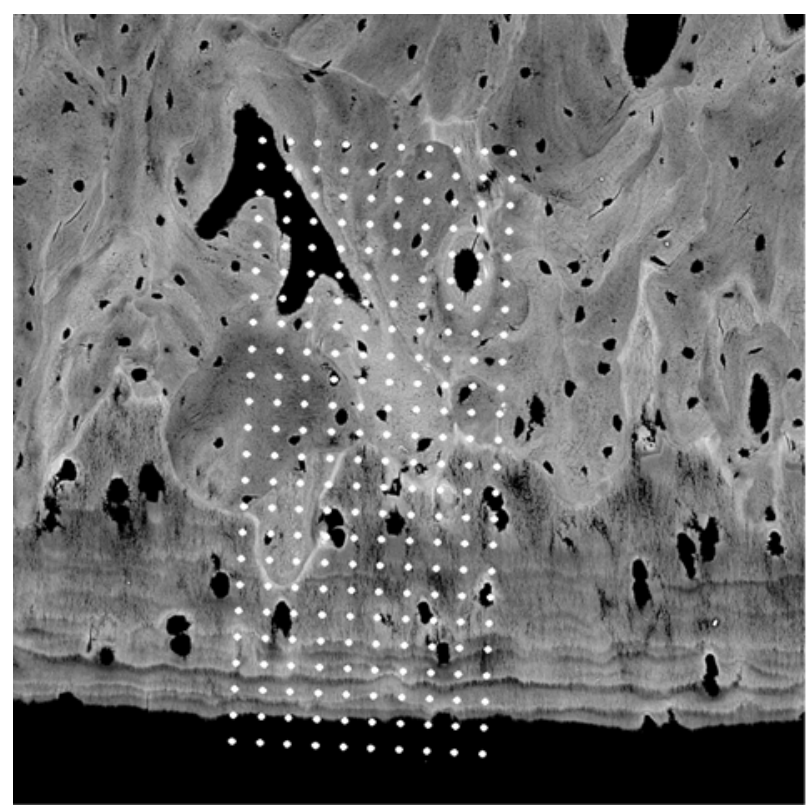

Figure 1. BSE SEM of the medial condylar field of the distal third metacarpal bone (Mc3) of trained horse 14 to illustrate location of indent arrays. Distal to bottom of image. $594 \mu \mathrm{m}$ wide field recorded after UMIS nanoindention. $20 \mu \mathrm{m}$ separation between indentation spots. The bottom two rows of indent spots overlie nonmineralised, hyaline articular cartilage. Articular calcified cartilage extends from the third to the seventh row up on the left, and to the tenth row on the right of the array, and above this is subchondral bone.

subchondral bone that we consider is a high turnover material and none of it persists for long enough to reach the degree of maturity of deeper epiphyseal bone: it is thus not highly mineralised (Boyde and Firth, 2005).

Fig. 1 shows a typical field covering a 10 wide nanoindentation array at $20 \mathrm{~mm}$ intervals calibrated according to the standards and with the nano-indentation sites superimposed as white dots. Site-matched values of elastic modulus $(E \mathrm{GPa})$ and mineralisation $(\mathrm{MVF} \%$ ) for calcified cartilage and bone for all the samples are shown in Figures 2 and 3.

Differences in normal loading levels are expected between trained and untrained horses, and the effect of the training and associated joint loading has been amply confirmed in prior studies of the specimens used in the present study. Thus there was an obvious increase in palmar condylar bone densification shown by peripheral computed tomographic imaging of the whole bones (Firth et al., 2005) and by light and scanning electron microscopic imaging techniques on sections of the same material (Boyde and Firth, 2005). In the present study, we examined only the calcified tissue layers closest to the joint surface exposed to this increased load, hypothesising that this tissue would have undergone significant adaptive change as a consequence. However, no difference was found (Table 1).

This lack of a demonstrable difference in compositional and biomechanical properties of mineralised tissue between trained and untrained animals is profoundly interesting, 
Table 1. The descriptive statistics for the Mean Volume Fraction (MVF) and the Elastic modulus (E) for the articular calcified cartilage (ACC) and the subchondral bone (SBC) for both the trained and untrained animals.

\begin{tabular}{|c|c|c|c|c|c|}
\hline & & \multicolumn{2}{|c|}{ Trained } & \multicolumn{2}{|c|}{ Untrained } \\
\hline & & $\begin{array}{l}\text { MVF } \\
\text { (\%) }\end{array}$ & $\begin{array}{l}\text { E } \\
\text { (GPa) }\end{array}$ & $\begin{array}{l}\text { MVF } \\
(\%)\end{array}$ & $\begin{array}{l}\text { E } \\
\text { (Gpa) }\end{array}$ \\
\hline \multirow[t]{4}{*}{ ACC } & Mean & 27.43 & 15.02 & 26.98 & 13.75 \\
\hline & SD & 6.90 & 1.65 & 7.17 & 2.22 \\
\hline & $\mathbf{n}$ & \multicolumn{2}{|l|}{458} & \multicolumn{2}{|l|}{565} \\
\hline & SEM & 0.32 & 0.08 & 0.30 & 0.09 \\
\hline \multirow[t]{4}{*}{$\begin{array}{l}\text { SCB } \\
\end{array}$} & Mean & 26.10 & 15.99 & 27.60 & 15.15 \\
\hline & SD & 5.49 & 1.72 & 5.21 & 2.06 \\
\hline & $\mathbf{n}$ & \multicolumn{2}{|l|}{448} & \multicolumn{2}{|l|}{736} \\
\hline & SEM & 0.26 & 0.081 & 0.19 & 0.08 \\
\hline
\end{tabular}

because it suggests that tissue structure and function was already optimised at normal levels of exercise in our two year old horses. Yet, both the articular calcified cartilage and the immediately adjacent subchondral bone transmit the excess loads associated with cantering and galloping. This, in the trained group, gives rise to large tissue responses deep within the condyles and even a significant increase in apparent bone mineral density of the third metacarpal diaphyseal cortical bone (Firth et al., 2005) which is more refractory to mechanical stimulation than cancellous bone. However, exercise levels of greater duration or intensity than those of the training regimen used in these horses here may be associated with pathologically high levels of mineralisation in both these thin tissue layers, and other factors such as limb conformation, neuromuscular function and track characteristics may also be involved.

The present study confirms our prior observations, using quantitative backscattered electron imaging, that there is a greater local variation in mineral concentration in cartilage than in bone (Figure 1). This reflects the presence of 'tidemarks', layers parallel with the mineralisation front with different degrees of calcification, some of these having higher levels than are normally found in bone, and others lower. Both of these observations support the view that there is no maturation mineralisation step in calcified cartilage, so that regions with lower mineral content in cartilage remain as such when buried beneath the mineralisation front, whereas maturation of bone packets occurs in subchondral bone.

\section{Acknowledgements}

We thank the Horserace Betting Levy Board and the New Zealand Racing Board for funding, and Maureen Arora and Roy Radcliffe for assistance with sample preparation.

\section{References}

Boyde A, Firth EC (2005) Musculoskeletal responses of 2-year-old thoroughbred horses to early training. 8. Quantitative back-scattered electron scanning electron microscopy and confocal fluorescence microscopy of the epiphysis of the third metacarpal bone. $\mathrm{N} Z \mathrm{Z}$ Vet $\mathrm{J}$ 53: $123-132$.

Bushby AJ (2001) Nano-indentation using spherical indenters. Non-Destructive Testing Evaluation 17:213234.

Bushby AJ, Jennett NM (2001) Determining the area function of spherical indenters for nanoindentation. Materials Res Soc Symp Proc 649: Q7.17.1-6.

Ferguson VL, Bushby AJ, Boyde A (2003) Nanomechanical properties and mineral concentration in articular calcified cartilage and subchondral bone. J Anat 203: 191-202.

Field JS, Swain MV (1993) A simple predictive model for spherical indentation. J Mat Res 8: 297-306.

Firth EC, Rogers CW (2005) Musculoskeletal responses of 2-year-old thoroughbred horses to early training. 7. Bone and articular cartilage response in the carpus. N Z Vet J 53: 113-122.

Firth EC, Rogers CW, Doube M, Jopson NB (2005) Musculoskeletal responses of 2-year-old thoroughbred horses to early training. 6 . Bone parameters in the third metacarpal and third metatarsal bones. N Z Vet J 53: 101112.

Firth EC, Rogers CW, Perkins NR, Anderson BH, Grace ND (2004) Musculoskeletal responses of 2-yearold thoroughbred horses to early training. 1. Study design, and clinical, nutritional, radiological and histological observations. N Z Vet J 52: 261-271.

Gupta HS, Schratter S, Tesch W, Roschger P, Berzlanovich A, Schoeberl T, Klaushofer K, Fratzl P (2005) Two different correlations between nanoindentation modulus and mineral content in the bone-cartilage interface. J Struct Biol 149: 138-148.

Howell PGT, Boyde A (1998a) Monte Carlo simulation of electron backscattering from compounds with low mean atomic number. Scanning 20: 45-49.

Howell PGT, Boyde A (1998b) Mean atomic number and backscattered electron coefficient calculations for some materials with low mean atomic number. Scanning 20: 35-40.

Howell PGT, Koole LH, Wetzels GMR, Aldenhoff YBJ, Boyde A (2001) The BSE signal from an iodinated polymer series: use for standardising BSE images for bone 

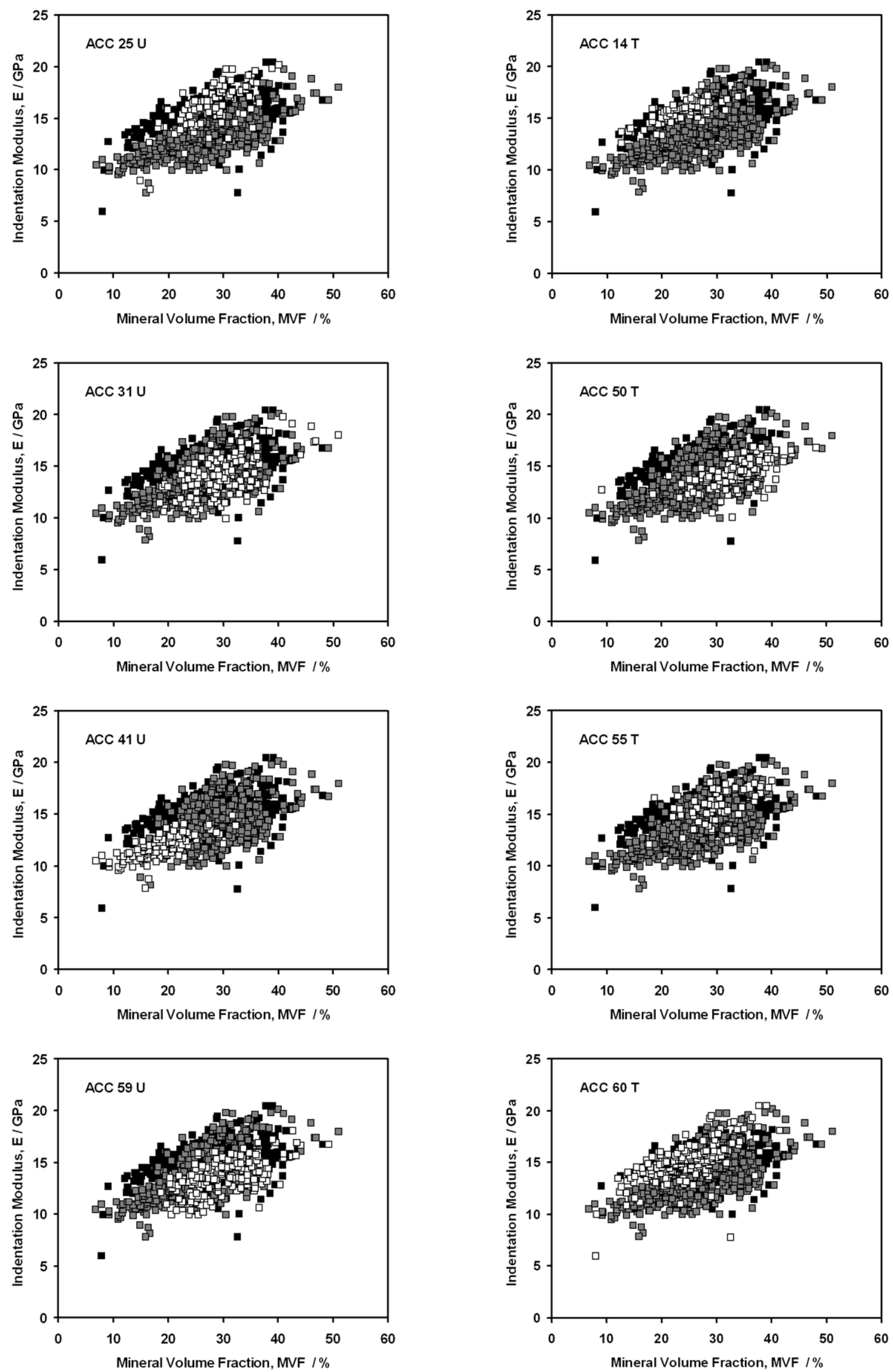

Figure 2

Figures 2 and 3. Plots of indentation modulus against mineral volume fraction for mineralised voxels for articular calcified cartilage (Figure 2) and subchondral bone (Figure 3). Grey = Untrained, Black = Trained animals, White symbols show the values for the individual horse identified in the figure. $\mathrm{ACC}=$ articular calcified cartilage. $\mathrm{SCB}=$ subchondral bone. $\mathrm{U}=$ Untrained horses numbers $25,31,41,59$ in left columns, $\mathrm{T}=$ Trained horses numbers 14,50 , 55,60 in right columns. 
VL Ferguson et al.
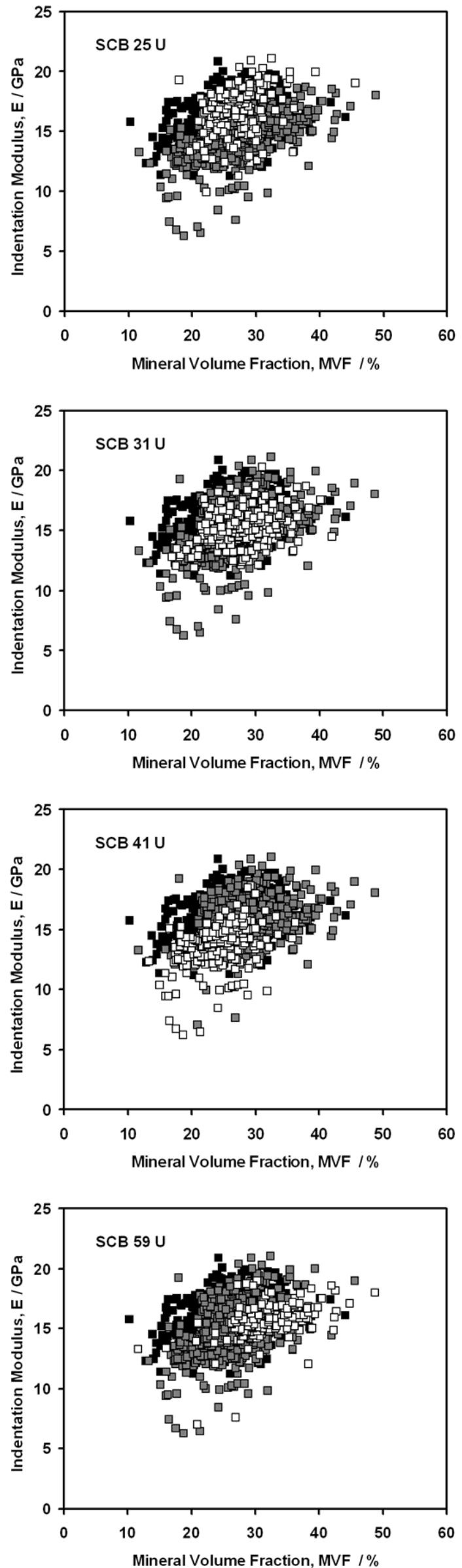

Figure 3
Training and calcified tissues in equine fetlock joint
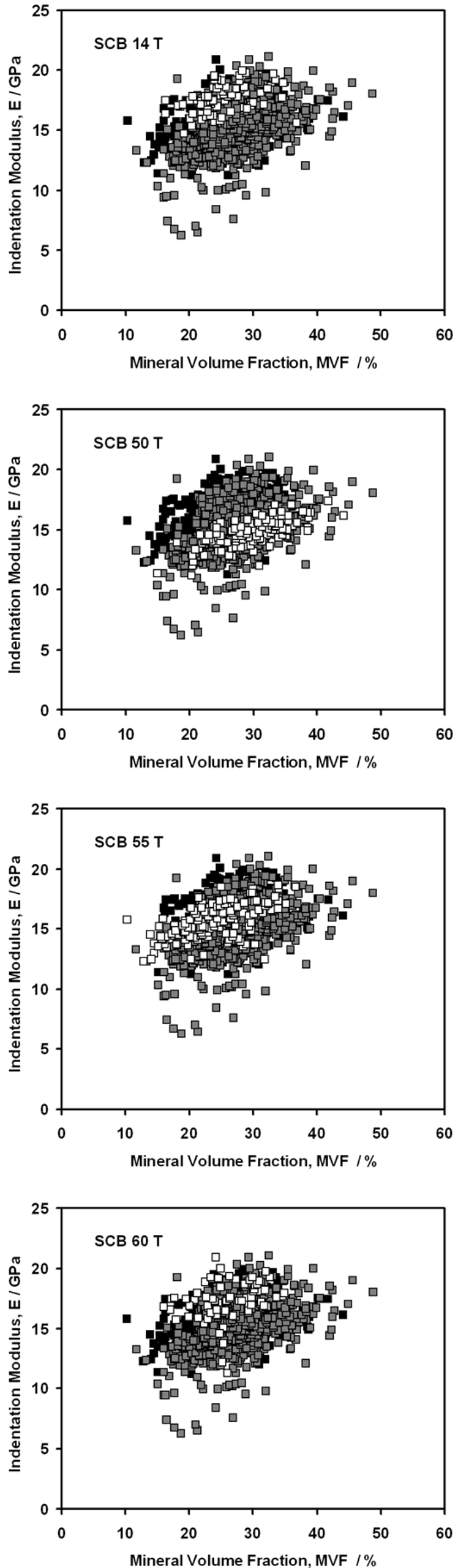
mineralisation density measurement. Scanning 23: 117118 .

Rogers CW, Firth EC (2004) Musculoskeletal responses of 2-year-old Thoroughbred horses to early training. 2. Measurement error and effect of training stage on the relationship between objective and subjective criteria of training workload. N Z Vet J 52: 272-279.

\section{Discussion with reviewers}

Reviewer 1: From a translational perspective what are the limitations of the current study to equine and human bone physiology?

Authors: The paper is presented because athletic training is common to man and some domestic animals, and in any species the training programme often does not meet the particular objective. The most common reason for this is musculo-skeletal injury, which precludes or reduces the possibility of exercise. In horses the most commonly afflicted tissue is articular cartilage, and the most common cause of lameness across breeds is osteoarthritis, which, as in people, causes pain and dysfunction. In horses, however, the progressive nature of the disease and the economic pressure on the species to perform can mean that some horses with chronic or severe OA must undergo treatment, spelling from work, and even disposal, all of which involve very high economic and welfare costs.

Reviewer 1: Given the extensive previous data (e.g., Boyde and Firth, 2005) it would be useful to have an additional statement regarding the difference and need for this current study.

Authors: The previous studies concentrated on the morphological effects of the earliest months of training on young thoroughbred racehorses, with no functional indication of the effect of that change. The present study sought to attach functional significance to the changes observed, in the very areas that had already been very carefully examined.

Reviewer 1: You provide a detailed and comprehensive exposition of the methods involved. The only minor issue is whether regions of interest examined are reproducible - given the previous publications - this is probably the case - but the authors could confirm this point.

Authors: The regions of interest are reproducible both globally, as between samples from different animals, and locally, as between different occasions of examining the same sites: in this latter case to micron scale precision on the block surface.

Reviewer 1: It is difficult given such small sample numbers (and trained Horse 60 refused to gallop) to accept that generalised statements are indeed valid as a general finding - a comment on this point would be useful.

and

Reviewer 2: It would be useful for the authors to consider and state where tissue structure and function is optimised in these large animals. It appears almost counter intuitive that the training required has no demonstrable effect on composition and biomedical properties of mineralised tissue between trained and untrained animals. What are the implications for the horse racing community and, are there implications for our understanding of the developmental origins of skeletal development?

Authors: We agree with Reviewer 2 that it is surprising that we found no differences between trained and untrained animals in the calcified cartilage layer of the joint that functions to attach soft cartilage to bone tissue proper. This is the more so considering that the differences in tissue adaptation within deeper tissue in the bone proper and in the same specimens were outstandingly obvious (Boyde and Firth, 2005), even in horse 60, as we already emphasised in the introduction. We therefore anticipated differences in the calcified cartilage, but they were not there. Thus whereas bone tissue is optimised consequential to 'training', calcified cartilage is already optimised at control levels of impact exercise. This is not to say that levels and duration of impact exercise above the training regime of the present experiment would not introduce detrimental effects which include excess mineralisation and fragmentation of articular calcified cartilage, which we believe to be the case.

As regards the number of animals, the reader should be aware that cost of purchase and maintenance is a major factor, and numbers have also to be kept as low as possible for ethical reasons. As above, the numbers were quite sufficient to demonstrate dramatic changes in bone in the same bones in the same animals.

As regards relevance to man, it is inconceivable that our results are not directly relevant to the human athlete or that we could perform such experiments and observations using human tissue. Actual loading levels will be more similar between man and horse than man and mouse or rat.

As regards relevance to the racing industry, we show two pieces of good news:- that normal levels of training exercise are beneficial by increasing the volumetric densification of bone (as reported in our earlier Boyde and Firth, 2005 paper) and have no detrimental effect within articular calcified cartilage.

With respect to developmental features of skeletal development, we have here only examined one intervention at one time point. Again, sensibly increased exercise was shown to increase bone volume fraction, but not to induce obvious changes in cartilage. We are pursuing studies of normal development of this joint at earlier time points.

Reviewer 1: Re Figures 2 and 3 - Could linear regression analysis provide any additional information?

Authors: No. We have shown that the correlation between elastic modulus and mineral volume fraction is normally very poor (Oyen et al., 2008) and so in this data it is not a good indicator of differences between exercised and nonexercised groups or indeed individuals within groups.

\section{Additional Reference}

Oyen ML, Ferguson VL, Bembey AK, Bushby AJ, Boyde A (2008) Composite Bounds on the Elastic Modulus of Bone. J Biomechanics 41: 2585-2588. 